\title{
Serum progranulin as an independent marker of liver fibrosis in patients with biopsy-proven nonalcoholic fatty liver disease
}

\author{
Yusuf Yilmaz ${ }^{\mathrm{a}, \mathrm{b}, *}$, Fatih Eren ${ }^{\mathrm{b}}$, Oya Yonal $^{\mathrm{a}}$, Zulfikar Polat ${ }^{\mathrm{c}}$, Mohammad Bacha ${ }^{\mathrm{d}}$, Ramazan Kurta ${ }^{\mathrm{a}, \mathrm{b}}$, \\ Oguzhan Ozturk ${ }^{\mathrm{e}}$ and Erol Avsar ${ }^{\mathrm{a}, \mathrm{b}}$ \\ ${ }^{a}$ Department of Gastroenterology, Marmara University, School of Medicine, Istanbul, Turkey \\ b Institute of Gastroenterology, Marmara University, Istanbul, Turkey \\ ${ }^{\mathrm{c}}$ Department of Gastroenterology, Gülhane Military Medical Academy, Ankara, Turkey \\ ${ }^{\mathrm{d}}$ Marmara University, School of Medicine, Istanbul, Turkey \\ e Department of Gastroenterology, Goztepe Education and Research Hospital, Istanbul, Turkey
}

\begin{abstract}
Background: Elevated progranulin levels are associated with visceral obesity, elevated plasma glucose, and dyslipidemia. Progranulin has not been previously investigated as a biomarker of nonalcoholic fatty liver disease (NAFLD). We sought to determine whether serum progranulin levels are altered in patients with biopsy-proven NAFLD and if they are associated with their clinical, biochemical, and histological characteristics.

Subjects and methods: We measured serum progranulin levels in 95 patients with biopsy-proven NAFLD and 80 age- and sex-matched controls. The potential associations between progranulin and the characteristics of NAFLD patients were examined by multiple linear regression analysis.

Results: Serum progranulin levels were significantly higher in NAFLD patients $(34 \pm 13 \mathrm{ng} / \mathrm{mL})$ than in controls $(28 \pm 7 \mathrm{ng} / \mathrm{mL}$, $P<0.001$ ). In NAFLD patients, serum progranulin levels were associated with lipid levels and the degree of hepatic fibrosis. After adjustment for potential confounders, serum progranulin remained an independent predictor of the degree of hepatic fibrosis in NAFLD patients $(\beta=0.392 ; t=2.226, P<0.01)$.

Conclusions: Compared with controls, NAFLD patients have higher serum progranulin concentrations, which are closely associated with lipid values and the extent of hepatic fibrosis.
\end{abstract}

Keywords: Progranulin, nonalcoholic fatty liver disease, enzyme-linked immunosorbent assay, hepatic fibrosis

\section{Introduction}

Nonalcoholic fatty liver disease (NAFLD) is the most common form of metabolic liver disease in Western countries. It comprises a disease spectrum ranging from simple steatosis to steatohepatitis (NASH) [1]. NAFLD is now recognized as the hepatic manifestation of metabolic syndrome [2], and its prevalence has been linked to a number of different metabolic abnor-

${ }^{*}$ Corresponding author: Yusuf Yilmaz, Institute of Gastroenterology, Marmara University, Maltepe, 34840, Istanbul, Turkey. Tel.: +90 533440 3995; Fax: +90 216688 6681; E-mail: yusufyilmaz@ uludag.edu.tr. malities (e.g., obesity, type 2 diabetes, hyperlipidemia, and hypertension) [3]. Due to the global epidemic of obesity and the potential progression to advanced liver disease, NAFLD has become an important emerging public health issue [4].

Progranulin is a widely expressed 593-amino acid glycoprotein encoded by a single gene on chromosome 17q21. Evidence suggests that progranulin plays a key role in a range of biological processes, including tumorigenesis, wound repair, neovascularization, inflammation, cell migration and mitosis, and cell survival [5, 6]. Interestingly, progranulin is not expressed in normal hepatocytes but its expression has been found to be increased in a mouse model of early diet-induced 
NASH [7]. In addition, the expression of progranulin has been identified in cultured primary and immortalized hepatic stellate cells [8]. Moreover, this molecule has been linked with the fibrosis response which follows tissue injury [9]. In a recent clinical study, Youn et al. [10] have reported that serum progranulin concentrations are significantly increased in individuals with type 2 diabetes and in obese subjects with predominant visceral fat accumulation. It is also noteworthy that progranulin levels correlate significantly with BMI, C-reactive protein levels, and total cholesterol [10]. Because the relationship between progranulin and NAFLD in human clinical studies remains unclear, we sought to examine the nature and the strength of the association between serum progranulin concentrations and the severity of liver histology in patients with biopsy-proven NAFLD.

\section{Materials and methods}

\subsection{Study participants}

A total of 95 patients with NAFLD (50 males and 45 females, mean age, $47 \pm 8$ years) and 80 healthy subjects (41 males and 39 females, mean age, 47 \pm 9 years) were enrolled in this study. The two groups were comparable for age and sex distribution. All NAFLD patients were consecutively seen at the hospital-based specialized outpatient clinics of the Department of Gastroenterology, Marmara University School of Medicine, over the past 12 months. All patients described here were enrolled in a larger research project aiming at identifying novel biomarkers of NAFLD using a "knowledge-based" approach [1113]. The "knowledge-based" approach relies on a direct understanding of the pathophysiological processes that underlie the development of NAFLD. It consists of biochemical assays aiming to assess attractive novel candidate markers informed by the biology of the disease process [14]. Inclusion criteria for this study comprised: (a) ultrasonographic evidence of steatosis grade 1 or higher; (b) absent-to-low alcohol consumption, i.e. $<20 \mathrm{~g} /$ day; (c) evidence of NAFLD on liver biopsy; (d) exclusion of viral B and C hepatitis, Wilson's disease, $\alpha 1$-antitrypsin deficiency, autoimmune hepatitis, genetic hemochromatosis, and use of steatogenic drugs. Patients were excluded when they had major comorbidities, i.e. ischemic heart disease, cerebrovascular diseases, autoimmune disorders, malignancies, and chronic renal insufficiency. A total of 80 healthy age- and sex-matched volunteers served as controls. All subjects included in the control group were judged to be in good health, with normal results on liver function tests, and confirmed as having normal liver by ultrasound. Subjects with a consumption of alcohol $>$ $20 \mathrm{~g} /$ day or who were taking any medication were not included in the control group. All patients and controls were of Turkish descent. The Ethics Committee of the Marmara University School of Medicine approved this study and all participants provided written informed consent.

\subsection{Clinical and biochemical characterization}

All subjects underwent a detailed physical examination, anthropometric measurements, and biochemical screening. Body mass index (BMI) was calculated from measurements of height and weight. Diabetes mellitus was diagnosed according to ADA criteria [15]. The metabolic syndrome was diagnosed using the ATP III criteria [16]. The estimate of insulin resistance was calculated using the HOMA-IR index, with the following formula: insulin resistance $=$ fasting plasma insulin (in microunits per milliliter) $\times$ fasting plasma glucose (FPG) (in millimoles per liter)/22.5. Blood pressure was measured using a mercury sphygmomanometer in a quiet room after $\geqslant 10$-min rest. Korotkoff 1 and 5 were taken for systolic blood pressure and diastolic blood pressure, respectively. Routine blood chemistry analyses were performed at the central laboratory of our hospital. Serum high-sensitivity C-reactive protein (hs-CRP) levels were measured in duplicate using a commercially available method (Dade Behring, Marburg, Germany). The intra-assay and the interassay coefficients of variation for hs-CRP were $4.6 \%$ and $6.1 \%$, respectively. The lower detection limit was $0.19 \mathrm{mg} / \mathrm{dL}$.

\subsection{Liver histology}

Ultrasonography-guided liver biopsies were performed under conscious sedation using a 16-gauge Hepafix needle (Braun Melsungen AG, Germany). All biopsy specimens were placed in formalin solution for fixation and embedded in paraffin blocks. Serial sections (sectioned at $4 \mathrm{~mm}$ intervals) were stained with hematoxylin-eosin, Masson's trichrome. An experienced pathologist blinded to clinical data scored the liver biopsies according to the NIDDK NASH Clinical Research Network scoring system [17]. Steatosis was scored from 0 to 3 with a four grades scoring system 
from S0 to S3: S0: no steatosis or less than 5\%, S1: 533\%, S2: 33-66\%, S3: >66\%. Lobular inflammation was graded as follows: stage 0 , no foci; stage 1: $<2$ foci per $200 \times$ field; stage 2 : $2-4$ foci per $200 \times$ field; stage 3: $>4$ foci per $200 \times$ field. Ballooning degeneration of liver cells was evaluated as: grade 0 , absent; grade 1, few cells; grade 2, many cells. The histological NASH score was defined as the unweighted sum of the scores for steatosis (0-3), lobular inflammation (0-3), and ballooning (0-2); thus ranging from 0 to 8 . Cases with scores of $0-2$ were considered as having simple steatosis; on the other hand, cases with scores of 5 or greater were diagnosed as definitive NASH. Cases with activity scores of 3 and 4 were considered as borderline NASH [17]. Fibrosis was staged as follows: stage 0: no fibrosis; stage 1: perisinusoidal or periportal fibrosis with 3 different patterns: 1A: mild, zone 3 , perisinusoidal; $1 \mathrm{~B}$ : moderate, zone 3 , perisinusoidal fibrosis, and $1 \mathrm{C}$ portal/periportal fibrosis; stage 2: perisinusoidal and portal/periportal fibrosis; stage 3 : bridging fibrosis; stage 4 : cirrhosis.

\subsection{Measurement of serum progranulin levels}

Blood samples were collected from an antecubital vein between 8:00 and 9.00 AM after an overnight fast. Samples were centrifuged at $2500 \mathrm{~g}$ for $10 \mathrm{~min}$, and serum aliquots were stored at $-80^{\circ} \mathrm{C}$ until immediately before analysis. Serum progranulin levels were measured using a commercially available enyzme-linked immunosorbent assay (ELISA) (Progranulin ELISA kit E103, Mediagnost, Reutlingen, Germany) according to the manufacturer's protocol. Absorbance at $450 \mathrm{~nm}$ was read by using a microtiter plate reader. The detection limit of the assay was $0.018 \mathrm{ng} / \mathrm{mL}$. The intraand inter-assay coefficients of variance were $4.2 \%$ and $5.8 \%$, respectively.

\subsection{Data analysis}

The Kolmogorov-Smirnov test was performed in all continuous variables to define the presence of normality. Gaussian variables are expressed as mean \pm standard deviation (SD), skewed data as medians and interquartile ranges, and categorical variables as counts. Skewed variables were logarithmically transformed to improve normality before statistical analysis and then back-transformed to their natural units for presentation in the text and tables. The Student's $t$-test was used to evaluate the differences between the two study groups in normally distributed continuous variables. When
Table 2

Histology findings of NAFLD patients

\begin{tabular}{lc}
\hline Characteristic & Value \\
\hline Histological steatosis & $2(1-3)$ \\
Lobular inflammation & $2(1-3)$ \\
Ballooning & $2(1-2)$ \\
NASH score & $5(4-7)$ \\
Fibrosis & $1(0-2)$ \\
\hline
\end{tabular}

Data are given as medians and interquartile ranges.

normality was not confirmed, the Mann-Whitney $U$ test was used. Correlations among variables were tested using the Spearman's correlation coefficient. Multivariable stepwise linear regression analyses were performed to identify independent predictors of the histological features of NAFLD (i.e., steatosis, lobular inflammation, hepatocye ballooning, liver fibrosis); the covariates included were progranulin and all variables listed in Table 1. We determined the sensitivity and specificity of serum progranulin concentration in predicting NAFLD at different cutoff values by the construction of a ROC curve (sensitivity plotted against 1specificity at different concentrations of progranulin). All calculations were performed using SPSS version 14.0 (SPSS, Inc., Chicago, IL, USA) and MedCalc 7.2 (Mariakerke, Belgium). A value of $P<0.05$ (twosided) was considered statistically significant.

\section{Results}

Table 1 shows the general characteristics of subjects with and without NAFLD. The two study groups did not differ in terms of age, sex, and HDL cholesterol. Body mass index, HOMA-IR, AST, ALT, systolic and diastolic blood pressure, total cholesterol, LDL cholesterol, and tryglicerides of NAFLD patients differed significantly from the control group. The prevalence of diabetes and the metabolic syndrome was higher in NAFLD patients than in controls. Table 2 summarizes the severity of histological features of NAFLD patients.

Serum progranulin levels were significantly higher in NAFLD patients $(34 \pm 13 \mathrm{ng} / \mathrm{mL})$ than in controls $(28$ $\pm 7 \mathrm{ng} / \mathrm{mL}$, Student's $t$ test, $P<0.001$, Fig. 1$)$. The ROC curve analysis (Fig. 2) suggested that the most useful cutoff value of serum progranulin concentration was $30 \mathrm{ng} / \mathrm{mL}$, where the sum of sensitivity (59\%) and specificity $(70 \%)$ was the highest.

We then analyzed the associations between serum progranulin levels and the clinical, biochemical, and histological characteristics of the study participants. In 
Table 1

General characteristics of the study participants

\begin{tabular}{lccc}
\hline & $\begin{array}{c}\text { NAFLD patients } \\
(n=95)\end{array}$ & $\begin{array}{c}\text { Control group } \\
(n=80)\end{array}$ & $P$ value \\
\hline Sex (males/females) & $50 / 45$ & $41 / 39$ & $\mathrm{NS}$ \\
Age (years) & $47 \pm 8$ & $47 \pm 9$ & $\mathrm{NS}$ \\
Body mass index (kg/m $\left.{ }^{2}\right)$ & $30.4 \pm 4.4$ & $27.6 \pm 4.1$ & $<0.01$ \\
Diabetes mellitus (yes/no) & $30 / 65$ & $0 / 80$ & $<0.001$ \\
Metabolic syndrome (yes/no) & $61 / 34$ & $0 / 80$ & $<0.001$ \\
HOMA-IR & $3.8(2.4-5.2)$ & $1.5(0.6-2.4)$ & $<0.001$ \\
Systolic blood pressure (mmHg) & $134 \pm 23$ & $122 \pm 18$ & $<0.01$ \\
Diastolic blood pressure (mmHg) & $84 \pm 12$ & $77 \pm 10$ & $<0.01$ \\
AST (U/L) & $46 \pm 16$ & $23 \pm 9$ & $<0.001$ \\
ALT (U/L) & $69 \pm 34$ & $20 \pm 9$ & $<0.001$ \\
Total cholesterol (mg/dL) & $223 \pm 52$ & $192 \pm 45$ & $<0.01$ \\
HDL cholesterol (mg/dL) & $44 \pm 12$ & $43 \pm 17$ & $\mathrm{NS}$ \\
LDL cholesterol (mg/dL) & $151 \pm 55$ & $124 \pm 19$ & $<0.001$ \\
Triglycerides (mg/dL) & $181 \pm 83$ & $143 \pm 69$ & $<0.001$ \\
hs-CRP (mg/dL) & $3.2(2.6-4.4)$ & - & - \\
\hline
\end{tabular}

HOMA-IR: homeostasis model of insulin resistance; AST: aspartate aminotransferase; ALT: alanine aminotransferase; HDL: high-density lipoprotein; LDL: lowdensity lipoprotein; hs-CRP: high-sensitivity C-reactive protein; NASH: nonalcoholic steatohepatitis. Data are given as means and SD, counts, or medians and interquartile ranges, as appropriate.

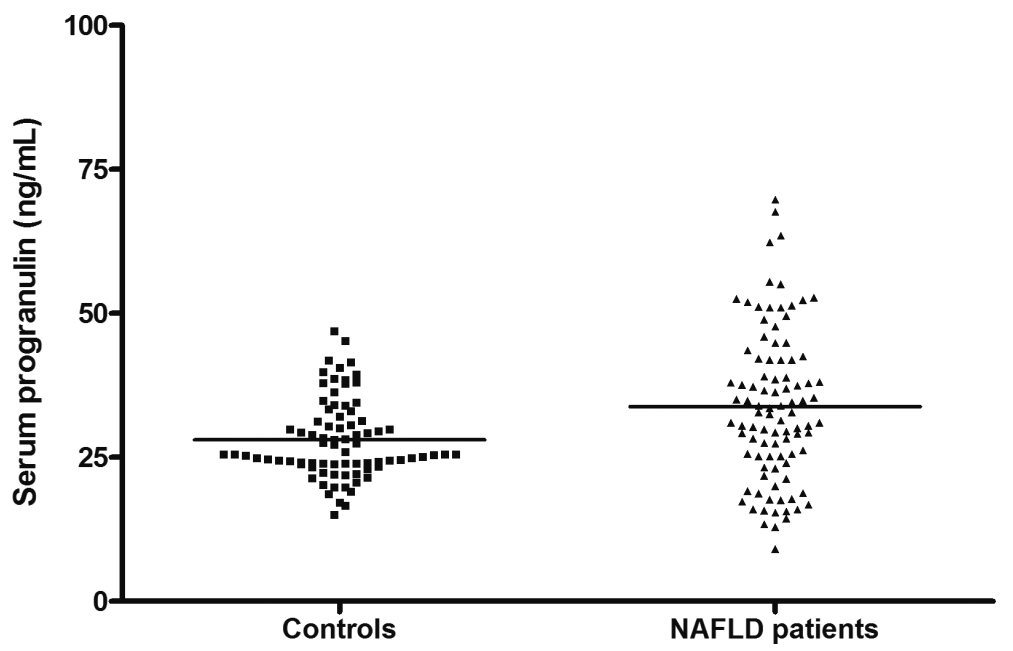

Fig. 1. Scatter diagram for serum progranulin levels in NAFLD patients and healthy controls. Horizontal lines across the scatter diagram represent mean values.

patients with NAFLD, serum progranulin levels were positively associated with total cholesterol $(r=0.38$, $P<0.001)$, LDL cholesterol $(r=0.32, P<0.001)$, and the degree of hepatocyte fibrosis $(r=0.22, P<$ $0.01)$. A serum progranulin level $>32 \mathrm{ng} / \mathrm{ml}$ had a positive predictive value for a fibrosis score $\geqslant 2$ of $93 \%$ and a negative predictive value of $56 \%$. There was no association between serum progranulin levels and the NASH score (data not shown).

After stepwise linear regression analysis adjusting for age, sex, body mass index, diabetes mellitus, the metabolic syndrome, HOMA-IR, blood pressure val- ues, liver enzymes, lipid variables, and hs-CRP, serum progranulin levels retained their independent significance as a predictor of hepatocyte fibrosis in NAFLD patients $(\beta=0.392 ; t=2.226, P<0.01)$.

\section{Discussion}

The role of progranulin in the pathogenesis of metabolic liver diseases is unclear due to the lack of the systematic observations. To our knowledge, this is the first study to examine the potential alterations 


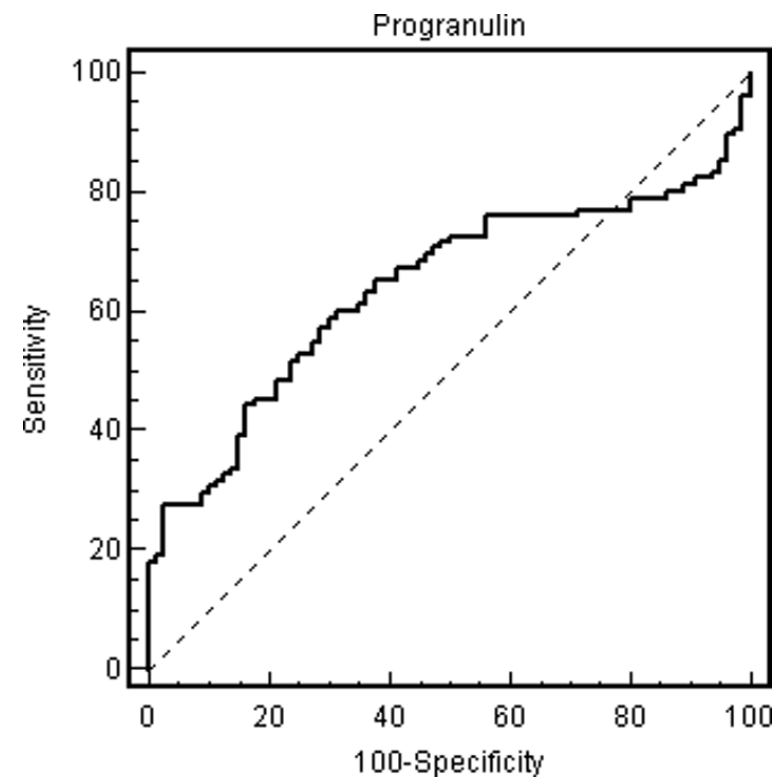

Fig. 2. ROC curve of serum progranulin concentration for the diagnosis of NAFLD. The area under the curve was 0.64 (standard error: 0.042 ; $95 \%$ confidence interval $0.57-0.71$ ).

of serum progranulin levels in patients with biopsyproven NAFLD. The results from this study indicate that (i) NAFLD patients have significantly higher serum progranulin concentrations than controls; (ii) progranulin concentrations are positively associated with serum lipid levels in NAFLD patients; and (iii) progranulin levels are independently associated with the degree of hepatic fibrosis among NAFLD patients after adjustment for a broad spectrum of potential confounders.

The selection of progranulin as a novel candidate biomarker for NAFLD is supported by the hyperexpression of this molecule in a mouse model of this condition [7], its expression in primary hepatic stellate cells [8], and its association with fibrosis following experimental injury [9]. In our study, serum progranulin levels were positively associated with total and LDL cholesterol. This relationship is intriguing as dyslipidemia is one of the core feature of the metabolic syndrome [16]. The results of the present study confirm and expand those of Youn et al. [10] who showed in a pilot study that serum progranulin levels are positively associated with total cholesterol. Another observation is that serum progranulin was independently associated with the extent of liver fibrosis in NAFLD patients. Of note, this relation was independent from serum lipid values in multivariable regression analysis. Although this was an observational clinical study and no direct inference can be made on the mechanisms linking altered levels of progranulin with hepatic fibrogenesis, we speculate that this can occur via the regulation of the hepatocyte growth factor (HGF)/mesenchymal-epithelial transition factor (c-Met) system signaling pathway. In this regard, a very recent study has shown that progranulin plays a key role in the regulation of this biochemical mechanism in vivo [18], which in turn has been shown to be linked with chronic liver injury and fibrosis progression in both humans and experimental models [19]. However, the precise mechanisms underlying the increase of progranulin in patients with NAFLD need further investigation. Since both the cellular source of serum progranulin and its mechanisms of secretion are multiple $[8,9]$, it is unclear whether the significant elevation of serum progranulin levels in NAFLD reflects a higher production or a reduced clearance. In this study, serum progranulin levels were related to the degree of fibrosis but not to the presence of NASH. This observation seems to suggest that progranulin can affect hepatic fibrosis but not the degree of steatosis or necroinflammation. We propose that the relationship between progranulin and fibrosis reflects a specific pathogenic role of progranulin in hepatic fibrogenesis and/or a failure of antifibrogenic pathways.

Several limitations of this study merit comment. First, our study has a cross-sectional nature and does not elucidate the causal relationship between serum progranulin levels and the presence of liver fibrosis. Therefore, we do not know whether serum progranulin levels could be mechanistically related to hepatic fibrosis by reflecting tissue progranulin expression in the liver. Eventually, a longitudinal study is needed to clarify the causal relationship between progranulin levels and liver injury. Second, the relatively small sample size limits the generalizability of our conclusions. Third, our sample included subjects of Turkish nationality, so that results cannot be extrapolated to populations with different ethnic background. These limitations notwithstanding, this is the first clinical study to demonstrate a relationship between progranulin and liver injury in patients with NAFLD. In addition, all patients with NAFLD underwent liver biopsy, the best standard for diagnosing this condition $[20,21]$. Finally, it is noteworthy that NAFLD patients with significant comorbidities were excluded from this study. Therefore, the confounding effects of concomitant diseases on the observed associations should not be so influential.

In conclusion, the results from this study demonstrate that patients with biopsy-proven NAFLD have 
significantly higher serum progranulin concentrations (vs. matched controls) that independently predict the extent of liver fibrosis. Further studies using larger populations will be needed to confirm our observations and to validate the current findings.

\section{Acknowledgements}

This study was supported by grants from the Institute of Gastroenterology, Marmara University. We are grateful to Prof. Dr. Cigdem Ataizi Celikel for her help in the assessment of liver biopsy specimens.

\section{References}

[1] S.K. Erickson, Nonalcoholic fatty liver disease, J Lipid Res 50 (2009), S412-S416.

[2] D.A. Sass, P. Chang and K.B. Chopra, Nonalcoholic fatty liver disease: a clinical review, Dig Dis Sci 50 (2005), 171-180.

[3] P. Almeda-Valdés, D. Cuevas-Ramos and C.A. AguilarSalinas, Metabolic syndrome and non-alcoholic fatty liver disease, Ann Hepatol 8 (2009), S18-S24.

[4] C.D. Byrne, R. Olufadi, K.D. Bruce, F.R. Cagampang and M.H. Ahmed, Metabolic disturbances in non-alcoholic fatty liver disease, Clin Sci (Lond) 116 (2009), 539-564.

[5] C.H. Ong and A. Bateman, Progranulin (granulin-epithelin precursor, PC-cell derived growth factor, acrogranin) in proliferation and tumorigenesis, Histol Histopathol 18 (2003), 1275-1288.

[6] Z.He and A. Bateman, Progranulin (granulin-epithelin precursor, PC-cell-derived growth factor, acrogranin) mediates tissue repair and tumorigenesis, J Mol Med $\mathbf{8 1}$ (2003), 600-612.

[7] R. Shiri-Sverdlov, K. Wouters, P.J. van Gorp, M.J. Gijbels, B. Noel, L. Buffat, B. Staels, N. Maeda, M. van Bilsen and M.H. Hofker, Early diet-induced non-alcoholic steatohepatitis in APOE2 knock-in mice and its prevention by fibrates, $J$ Hepatol 44 (2006), 732-741.

[8] L. Xu, A.Y. Hui, E. Albanis, M.J. Arthur, S.M. O’Byrne, W.S. Blaner, P. Mukherjee, FS.L. Riedman and F.J. Eng, Human hepatic stellate cell lines, LX-1 and LX-2: new tools for analysis of hepatic fibrosis, Gut 54 (2005), 142-151.

[9] Z. He, C.H. Ong, J. Halper and A. Bateman, Progranulin is a mediator of the wound response, Nat Med 9 (2003), 225-229.

[10] B.S. Youn, S.I. Bang, N. Klöting, J.W. Park, N. Lee, J.E. Oh, K.B. Pi, T.H. Lee, K. Ruschke, M. Fasshauer, M. Stumvoll and
M. Blüher, Serum progranulin concentrations may be associated with macrophage infiltration into omental adipose tissue, Diabetes 58 (2009), 627-636.

[11] Y. Yilmaz, F. Eren, O. Yonal, R. Kurt, B. Aktas, C.A. Celikel, O. Ozdogan, N. Imeryuz, C. Kalayci and E. Avsar, Increased serum FGF21 levels in patients with nonalcoholic fatty liver disease, Eur J Clin Invest 40 (2010), 887-892.

[12] Y. Yilmaz, O. Yonal, R. Kurt, Y.O. Alahdab, F. Eren, O. Ozdogan, C.A. Celikel, N. Imeryuz, C. Kalayci and E. Avsar, Serum levels of omentin, chemerin and adipsin in patients with biopsy-proven nonalcoholic fatty liver disease, Scand $J$ Gastroenterol 46 (2011), 91-97.

[13] Y. Yilmaz, O. Yonal, R. Kurt, F. Ari, A.Y. Oral, C.A. Celikel, S. Korkmaz, E. Ulukaya, O. Ozdogan, N. Imeryuz, E. Avsar and C. Kalayci, Serum fetuin A/ $\alpha 2 \mathrm{HS}$-glycoprotein levels in patients with non-alcoholic fatty liver disease: relation with liver fibrosis, Ann Clin Biochem 47 (2010), 549-553.

[14] Y. Yilmaz, A.E. Kedrah and O. Ozdogan, Cytokeratin-18 fragments and biomarkers of the metabolic syndrome in nonalcoholic steatohepatitis, World J Gastroenterol 15 (2009), 43874391.

[15] ACE/ADA Task Force on Inpatient Diabetes, American College of Endocrinology and American Diabetes Association Consensus statement on inpatient diabetes and glycemic control, Diabetes Care 29 (2006), 1955-1962.

[16] S.M. Grundy, H.B. Brewer, Jr., J.L. Cleeman, S.C. Smith, Jr. and C. Lenfant, American Heart Association; National Heart, Lung, and Blood Institute, Definition of metabolic syndrome: Report of the National Heart, Lung, and Blood Institute/American Heart Association conference on scientific issues related to definition, Circulation 109 (2004), 433-438.

[17] D.E. Kleiner, E.M. Brunt, M. Van Natta, C. Behling, M.J. Contos, O.W. Cummings, L.D. Ferrell, Y.C. Liu, M.S. Torbenson, A. Unalp-Arida, M. Yeh, A.J. McCullough and A.J. Sanyal, Design and validation of a histological scoring system for nonalcoholic fatty liver disease, Hepatology 41 (2005), 1313-1321.

[18] Y.H. Li, M.H. Chen, H.Y. Gong, S.Y. Hu, Y.W. Li, G.H. Lin, C.C. Lin, W. Liu and J.L Wu, Progranulin A-mediated MET signaling is essential for liver morphogenesis in zebrafish, $J$ Biol Chem 285 (2010), 41001-41009.

[19] T. Cramer, D. Schuppan, M. Bauer, D. Pfander, P. Neuhaus and $\mathrm{H}$. Herbst, Hepatocyte growth factor and c-Met expression in rat and human liver fibrosis, Liver Int 24 (2004), 335-344.

[20] E. Emanuele, Is biopsy always necessary? Toward a clinicolaboratory approach for diagnosing nonalcoholic steatohepatitis in obesity, Hepatology 48 (2008), 2086-2087.

[21] Y. Yilmaz and E. Dolar, Biomarkers for noninvasive biochemical diagnosis of nonalcoholic steatohepatitis: tools or decorations? World J Gastroenterol 15 (2009), 4346-4347. 


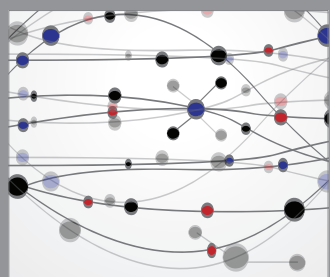

The Scientific World Journal
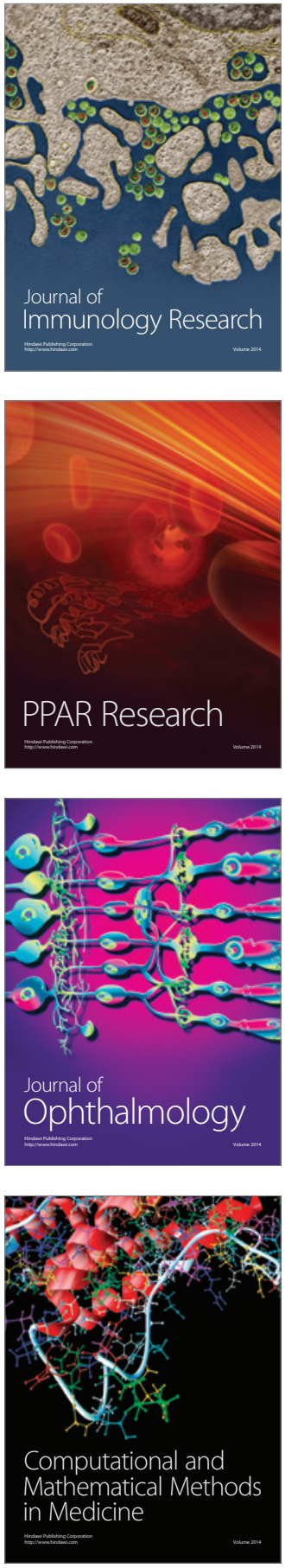

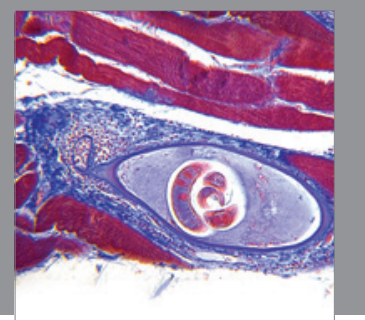

Gastroenterology

Research and Practice
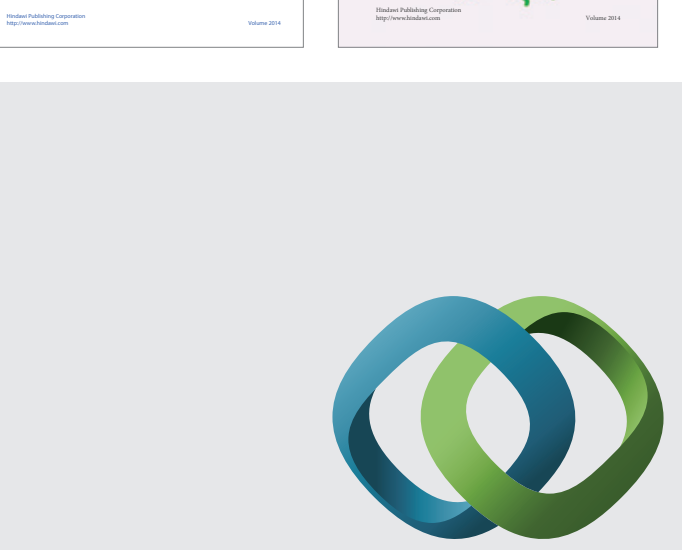

\section{Hindawi}

Submit your manuscripts at

http://www.hindawi.com
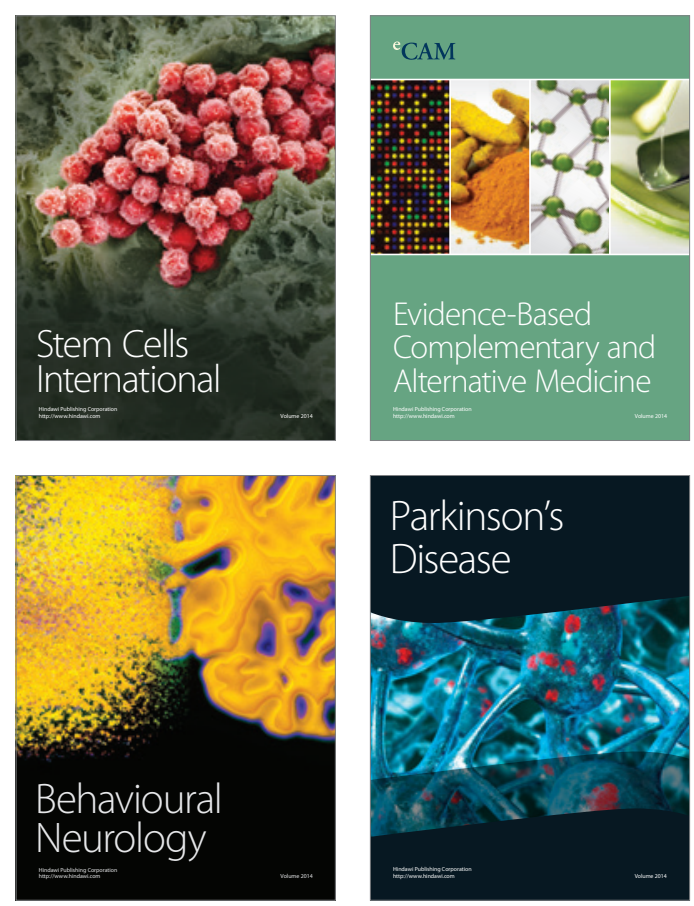

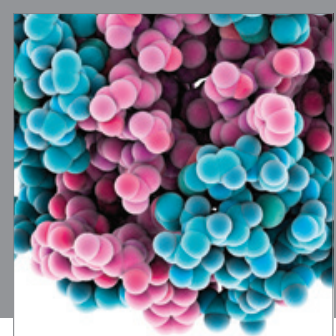

Journal of
Diabetes Research

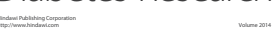

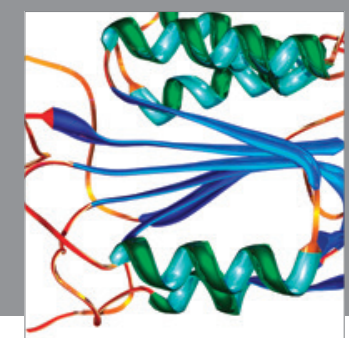

Disease Markers
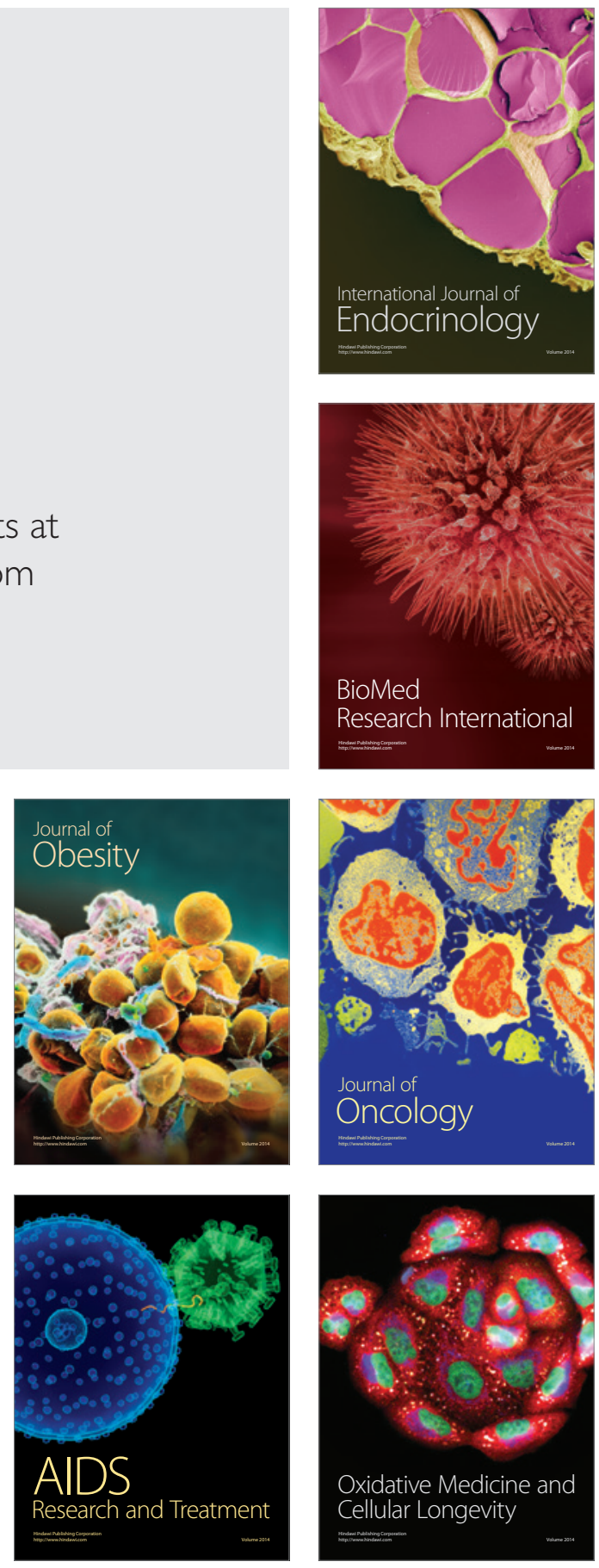